\title{
Additional Results to The struCtural ANALYSis OF ECCENTRICALLY COMPRESSED CROSS-SECTION MADE OF NO TENSION MATERIAL
}

\begin{abstract}
Máté Hazay
Institute of Civil Engineering, Ybl Miklós Faculty of Architecture and Civil Engineering, Szent István University, Budapest, Hungary matehazay@hotmail.com

Abstract: The mechanical behaviour of eccentrically compressed stocky bars of rectangular cross section, having no-tension material is well known. The aim of the structural analysis is to determine the distribution of the stresses arisen from the effect of an eccentrically compressive force, i.e. to describe the shape of the stress block, and to calculate the compressive resistance of the bar. Some important details of the phenomenon could not be found in the technical literature. In our paper we study these details. We evaluate the analytical functions of the curves, bounding areas lain around the kern or the point of gravity, on which areas the eccentric force acts a pentagonal compressed zone arisen. Furthermore, we determine the resistance of the stocky bar in the cases of triangular and rectangular compressive zone.
\end{abstract}

Keywords: eccentric compression, rectangular cross section, stocky bars.

\section{INTRODUCTION}

In elastic state at the four sides of the kern we can determine areas of three edges, bounded by the side of the kern and two curves, on which a compressive force acts, the shape of the compressed zone is a pentagon. We aim at providing the analytical functions of the curves bounding the area. Moreover, we give the shape of the stress block and its resultant - which supply the magnitude of the resistance of the bar - in case of triangular and rectangular compressed zone.

In plastic state the phenomenon of the kern disappears and only the role of the point of gravity remains. Then, stretching from the point of gravity into four directions lanceolate areas bounded with two curves could be given. The compressive force acting at this area causes a pentagonal compressed zone. We strive to evaluate the analytical functions of the curves bounding the area. Further, we determine the shape of the stress body and its resultant in case of triangular and rectangular compressed zone. 


\section{MY RESULTS}

\subsection{Analysis of the elastic state}

In course of the elastic analysis of an eccentrically compressed stocky bar, having material that resists only compression and having rectangular cross section, regarding mechanical behaviour, three areas of the possible locations of the point of action could be specified which considerably differ from each other: the force acts on the area of the kern (with the edge of the kern together) or it lies between the edge of the kern and the edge of the cross section (without the edges) or it is at the edge of the cross section or outside it. When eccentricity of the axial force is small (the load lies within the kern) the normal stresses will have the same sign throughout the cross section (in our case it has a minus sign i.e. the force is compressive). A condition of this kind is important, for instance, when a compressive load acts on the material, which is only suitable resisting compression, such as concrete, stone or masonry materials. For such materials, it may be necessary to ensure that the load produces no tension at any point of the cross section. The kern, regarding the mechanical behaviour is a bounded region, i.e. when compressive force acts at a point of its edge, the mechanical behaviour is the same as, if the force acts at an inside point of the kern. Some additional details about the analysis of the middle kern can be found in $[1,2,3]$. When the eccentric force acts outside the kern, but inside the edge of the cross section, only a region of the cross section is compressed, and three areas of the cross section could be determined, having different mechanical behaviour in eccentric compression (Figure 1). This figure can be found in [4].

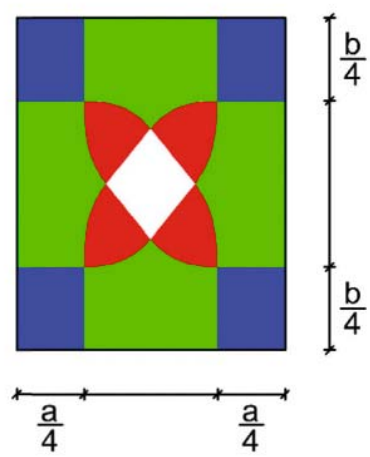

Figure 1 The different parts of the cross section

This is an open region, because, when the force acts at its edges, the mechanical behaviour differs from the behaviour as if, the force acts on the inside region.

Eccentric forces acting on the cross section, depending on the location of their point of action produce compressive zones of different shape. If the force acts at the outside quarters, close to the corners, the shape of the compressed zone is a right-angled triangle (blue shaded). This issue is analysed in [5]. A trapezoidal compressed zone belongs to those regions, which are outside of the curves and the rectangular parts (green shaded). The curves separate these previously mentioned areas from the zones, where the load would cause a pentagonal compressed zone (red shaded). The position of the dividing straight lines is already known, but we could not find the functions of the curves in the literature. 
That is why the first part of the elastic analysis of this essay is the analytic determination of curves, limiting the arched-shape regions, fitted to the kern. Having got the parameters of the location of the eccentric force and the formulae deduced of the compressed zone in this present essay, the resistance of the eccentrically loaded stocky bar having material that resists only compression, and having rectangular cross section could be determined as the volume of the stress body of linearly varying height. At one of the corner of the stress body (a truncated pyramid) the value of the stress is the yielding stress. In elastic state, when the greatest stress value does not reach the value of the yielding stress, the calculation could be made even in an inverse way. Taking the value of the eccentric force, the greatest corner stress of the stress body could be determined.

For the analysis of the curves, it is essential to know the sizes of the stress block which belongs to the simpler case of the trapezoid compressed zone (Figure 2, green shaded area). Getting the sizes of the trapezoid compressed zone, the volume of the stress block, which is equal to the load bearing capacity of the stocky bar, could be calculated.

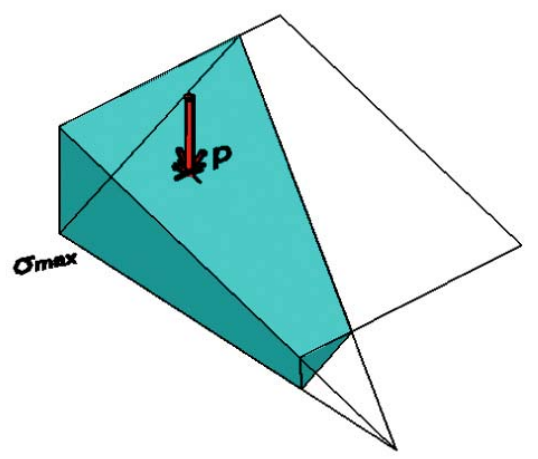

Figure 2 The stress body

During the calculation a coordinate system was used, which has the origin at the more compressed corner of the cross section (Figure 3). The stress block would be determined if we can calculate the length of the " $x$ " and " $y$ " edges of the pyramid.

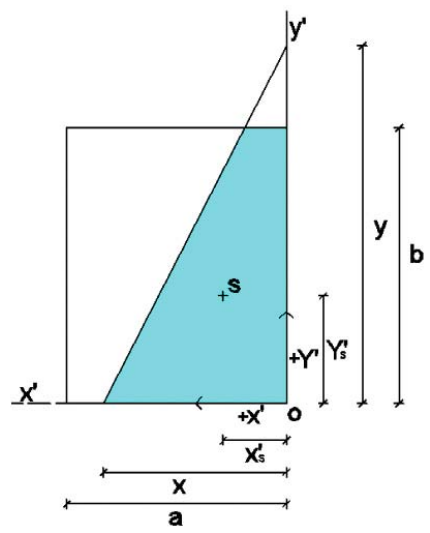

Figure 3 The coordinate system 
The same method can be used when we determine the place of the centre of gravity, but this time a reversed procedure should be applied. In this case the place of the centre of the gravity - which is the point, where the load acts - is given but the sizes of the body aren't known. As we can see in Figure 2, the stress body is a truncated pyramid, because a small part, which is also a pyramid, exceeds the cross section. We can handle this object with subtracting the volume of the small pyramid from the whole, big one. There are two unknowns, so two algebraically independent equations are needed to provide solution, therefore we will have to use this calculation method referring " $x$ " and " $y$ " directions as well. Let's start with the " $y$ " direction. In Eq. (1) the principle of the calculation can be seen.

$$
Y_{s}^{\prime}=\frac{S_{x}^{\prime}}{V}
$$

where:

$Y_{S}^{\prime} \quad-\quad$ Y coordinate of the point of action;

$S_{x}^{\prime} \quad-$ moment of stresses about the x' axis;

$V \quad-$ stress resultant.

In Eq. (2) we can see the initial equation.

$$
Y_{S}^{\prime}=\frac{\frac{x \cdot y \cdot \sigma \cdot y}{2 \cdot 3 \cdot 4}-\frac{(y-b) \cdot\left(x-\frac{b \cdot x}{y}\right) \cdot\left(\sigma-\frac{\sigma \cdot b}{y}\right) \cdot\left(b+\frac{y-b}{4}\right)}{2 \cdot 3}}{\frac{x \cdot y \cdot \sigma}{2 \cdot 3}-\frac{(y-b) \cdot\left(x-\frac{b \cdot x}{y}\right) \cdot\left(\sigma-\frac{\sigma \cdot b}{y}\right)}{2 \cdot 3}},
$$

where:

$\sigma \quad-$ quantity of the maximum normal stress

From Eq. (2), "y" can be expressed which is one of our unknowns. It is shown in Eq. (3).

$$
y=\frac{-6 \cdot b \cdot Y_{S}^{\prime}+4 \cdot b^{2}+b \cdot \sqrt{\left(-2 \cdot b^{2}+12 \cdot b \cdot Y_{S}^{\prime}-12 \cdot Y_{S}^{\prime 2}\right)}}{6 \cdot b-12 \cdot Y_{S}^{\prime}}
$$

Afterwards, we should follow the previous procedure referring to " $\mathrm{x}$ " direction too. The initial equation can be seen in Eq. (4). 


$$
X_{S}^{\prime}=\frac{\frac{x \cdot y \cdot \sigma \cdot x}{2 \cdot 3 \cdot 4}-\frac{(y-b) \cdot\left(x-\frac{b \cdot x}{y}\right) \cdot\left(\sigma-\frac{\sigma \cdot b}{y}\right) \cdot\left(x-\frac{b \cdot x}{y}\right)}{2 \cdot 3 \cdot 4}}{\frac{x \cdot y \cdot \sigma}{2 \cdot 3}-\frac{(y-b) \cdot\left(x-\frac{b \cdot x}{y}\right) \cdot\left(\sigma-\frac{\sigma \cdot b}{y}\right)}{2 \cdot 3}}
$$

where:

$X_{S}^{\prime} \quad-\quad \mathrm{X}$ coordinate of the point where the force acts.

From this starting equation " $\mathrm{x}$ " can be expressed which is the second unknown. It is shown in Eq. (5).

$$
x=\frac{6 \cdot y^{3} \cdot X_{S}^{\prime}-6 \cdot y^{2} \cdot b \cdot X_{S}^{\prime}+2 \cdot y \cdot b^{2} \cdot X_{S}^{\prime}}{2 \cdot y^{3}-3 \cdot y^{2} \cdot b+2 \cdot y \cdot b^{2}-\frac{b^{3}}{2}}
$$

Both unknowns have been already expressed, so substituting the right parameters into the equations, the sizes of the stress body can be determined. As we know, the resistance is equal to the volume of the stress body, therefore the resistance of the stocky element can be calculated. The resistance is expressed in Eq. (6).

$$
V=\frac{b \cdot x \cdot f_{y}}{2} \cdot\left(1-\frac{b}{y}+\frac{b^{2}}{3 \cdot y^{2}}\right)
$$

where:

$V \quad$ - volume of the stress body, which is the resistance at the same time;

$f_{y} \quad-$ yielding compressive strength of the material of the cross section.

This calculation method belongs to the point of action of the external force, when the shape of the compressed zone is a trapezoid. Actually, when the compressive force acts on the curve that is an ultimate situation, because the compressed zone is still a trapezoid, but exceeding the curve and arriving to the area between the arcs, the shape of the compressed zone is already a pentagon (Figure 4, 5). 


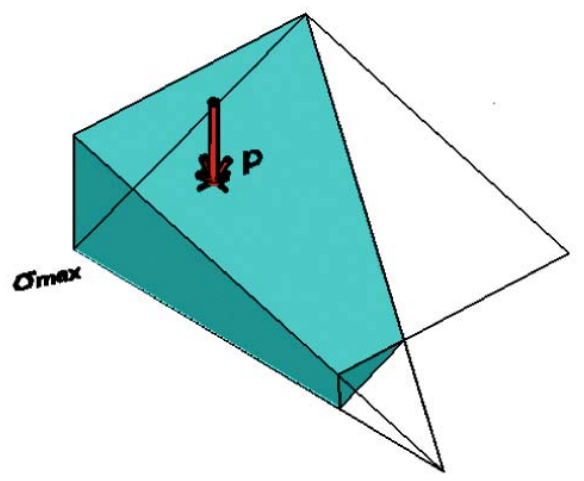

Figure 4 The stress body when the force acts on the curve

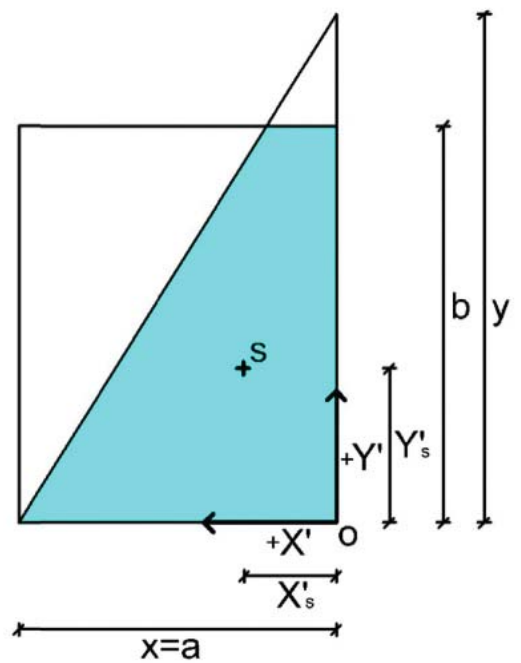

Figure 5 The shape of the compressed zone when the force acts on the curve

Thus, the previous equations can be used, assuming that " $\mathrm{x}$ " - which was an unknown quantity earlier - is equal to the length of the "a" edge of the cross section. This is shown in Eq. (7).

$$
x=\frac{6 \cdot y^{3} \cdot x_{s}^{\prime}-6 \cdot y^{2} \cdot b \cdot x_{s}^{\prime}+2 \cdot y \cdot b^{2} \cdot x_{s}^{\prime}}{2 \cdot y^{3}-3 \cdot y^{2} \cdot b+2 \cdot y \cdot b^{2}-\frac{b^{3}}{2}}=a
$$

From Eq. (7), " $X_{S}^{\prime \prime}$ can be expressed, which means that the curve has been determined, because in every " $Y_{S}^{\prime \prime}$ point, the other, " $X_{S}^{\prime}$ " coordinate of the point of the curve can be calculated (Figure 6). 


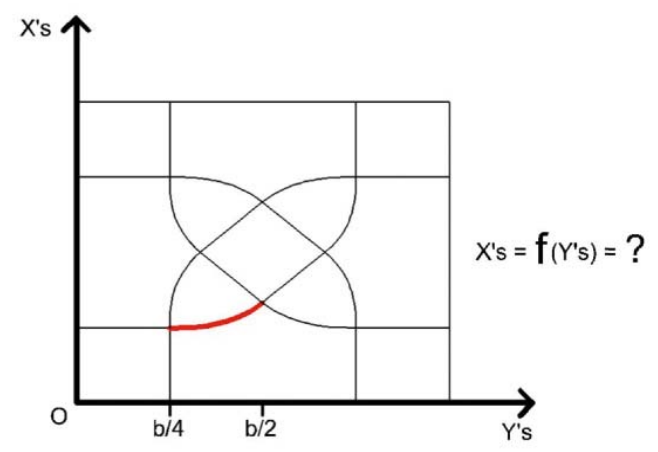

Figure 6 The graph of the function

The result which is the equation of the curve is shown in Eq. (8).

$$
X_{S}^{\prime}=\frac{a \cdot\left(2 \cdot y^{3}-3 \cdot y^{2} \cdot b+2 \cdot y \cdot b^{2}-\frac{b^{3}}{2}\right)}{6 \cdot y^{3}-6 \cdot y^{2} \cdot b+2 \cdot y \cdot b^{2}}
$$

In this present essay we made the analysis for compressed zones of rectangle triangle and quadratic shape. Analysis referring parameters of the compressed zone of pentagon shape could be the theme of further research.

\subsection{Analysis of the plastic state}

In course of the plastic analysis of an eccentrically compressed stocky bar, having material that resists only compression and having rectangular cross section, regarding mechanical behaviour, two area of the possible location of the point of action could be specified which considerably differ from each other: the force lies inside of the cross section (without the edge) or it is outside the cross section (with the edge). In plastic state regarding mechanical behaviour the cross section has no kern i.e. the kern degenerates into a point (it is the centroid of the cross section). Force acting at the centroid of the cross section produces simple, axial, centric compression of constant stress distribution. When the eccentric force acts outside the centroid, but inside the edge of the cross section, only a region of the cross section is compressed, and three areas of the cross section could be determined, having different mechanical behaviour (Figure 7). This figure can be found in $[6,7,8]$. Compressive force acting within this region produces only compression of uniform stress distribution. 


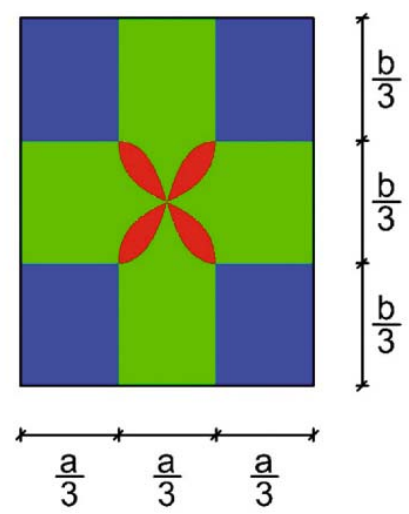

Figure 7 The different parts of the cross section

This is an open region, because the force acting at its edges, the mechanical behaviour considerably differs from the behaviour the force acting on the inside region. Eccentric forces acting on the cross section, depending on the location of their point of action produce compressive zones of different shape. If the force acts at the outside thirds, close to the corners (blue shaded), the shape of the compressed zone is rectangle triangle. A trapezoid compressed zone belongs to those regions, which are outside of the curves and the rectangular parts (green shaded). The curves separate these previously mentioned areas from the zones, where the load would cause a pentagonal compressed zone (red shaded). The position of the dividing straight lines is already known. But we could not find the functions of the limiting curves of the regions of arched-shape, fitted to the centroid in the literature.

That is why the first part of the plastic analysis of this essay is the analytic determination of curves, limiting the arched-shape regions, fitted to the centroid. Having got the parameters of the location of the eccentric force and the formulae deduced of the compressed zone below the resistance of the eccentrically loaded stocky bar having material that resists only compression, and having rectangular cross section could be determined as the volume of the stress body of constant height, which is the yielding stress.

During the calculation we can use the same method as we did previously, and we keep the same symbols and coordinate system, which are shown in Figure 3. We are dealing with a very similar situation, when the compressed zone is a trapezoid like earlier, but this time, due to the uniformly stress distribution the stress body is a prism which is depicted in Figure 8.

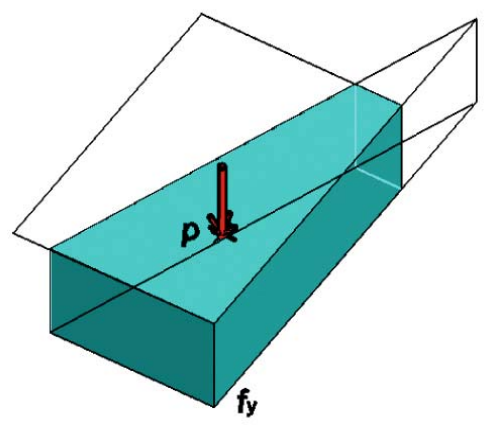

Figure 8 The stress body in plastic state 
At first, the sizes of the stress block should be determined. For that, we can use the principle of calculation method of the gravity centre again. Apparently, when the compressed zone is a trapezoid, then the stress body is a truncated prism. With subtraction the small triangle-based prism, which is out of the cross section, from the bigger right-angled triangle based prism the truncated prism can be described. Using this method and the previous symbols, the starting equation of the calculation in $\mathrm{Y}^{\prime}$ direction can be seen in Eq. (9).

$$
Y_{S}^{\prime}=\frac{\frac{x \cdot y \cdot y}{2 \cdot 3}-\frac{(y-b) \cdot\left(x-\frac{b \cdot x}{y}\right) \cdot\left(b+\frac{y-b}{3}\right)}{2}}{\frac{x \cdot y}{2}-\frac{(y-b) \cdot\left(x-\frac{b \cdot x}{y}\right)}{2}}
$$

As a result, the "y" side of the bigger right-angled triangle can be expressed from the previous equation, which is one of our unknowns. The formula of the " $y$ " size is shown in Equation 10.

$$
y=\frac{2 \cdot b^{2}-3 \cdot b \cdot Y_{S}^{\prime}}{3 \cdot b-6 \cdot Y_{S}^{\prime}}
$$

Afterwards, using the principle of the gravity centre calculation in the $X^{\prime}$ direction too, the other, " $\mathrm{x}$ " leg of the right-angled triangle can be determined. The starting equation according to the previous type of calculation is shown in Eq. (11).

$$
X_{S}^{\prime}=\frac{\frac{x \cdot y \cdot x}{2 \cdot 3}-\frac{(y-b) \cdot\left(x-\frac{b \cdot x}{y}\right) \cdot\left(x-\frac{b \cdot x}{y}\right)}{2 \cdot 3}}{\frac{x \cdot y}{2}-\frac{(y-b) \cdot\left(x-\frac{b \cdot x}{y}\right)}{2}}
$$

From Eq. (11) the " $x$ " leg of the big right-angled triangle, which is illustrated in Figure 3, can be expressed. The formula of the "x" size is shown in Eq. (12).

$$
x=\frac{2 \cdot X_{s}^{\prime} \cdot y^{2}-X_{s}^{\prime} \cdot b \cdot y}{y^{2}-b \cdot y+\frac{b^{2}}{3}}
$$

Both of our unknowns have already been expressed, thus the volume of the stress body can be calculated, therefore we can assess the resistance of the stocky bar because it is equal to the volume of the stress block. This formula is shown in Eq. (13).

$$
V=f_{y} \cdot b \cdot x \cdot\left(1-\frac{b}{2 \cdot y}\right)
$$

where: 
$V \quad-$ volume of the stress body, which is the resistance at the same time;

$f_{y} \quad-$ yielding compressive strength of the material of the cross section.

For the determination of the curve the same assumption can be used like earlier. Namely " $x$ " is not an unknown any more, but it is equal to the "a" size of the cross section. This substitution is shown in Eq. (14).

$$
x=\frac{2 \cdot x_{s}^{\prime} \cdot y^{2}-x_{s}^{\prime} \cdot b \cdot y}{y^{2}-b \cdot y+\frac{b^{2}}{3}}=a
$$

After the usage of the previous substitution the " $X_{S}^{\prime}$ " coordinate of that point of the curve can be determined which belongs to the particular " $Y_{S}^{\prime}$ " place.

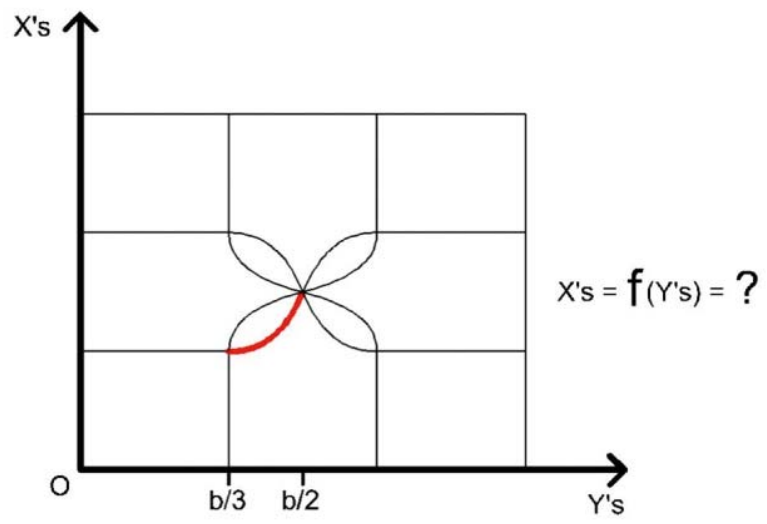

Figure 9 The analysis of the function

The expression of the " $X_{S}^{\prime}$ " coordinate, which is actually the equation of the curve is shown in Eq. (15).

$$
X_{S}^{\prime}=\frac{a \cdot\left(b^{2}-3 \cdot b \cdot Y_{s}^{\prime}+3 \cdot Y_{s}^{\prime 2}\right)}{2 \cdot b^{2}-3 \cdot b \cdot Y_{s}^{\prime}}
$$

In this present essay I made the analyses for compressed zones of rectangle triangle and quadratic shape. Analysis referring parameters of the compressed zone of pentagon shape could be the theme of further research. 


\section{CONCLUSION}

Using formulae evaluated of the above discussed structural engineering phenomenon, the structural designer can easily decide which compressed zone shape belongs to the given eccentricity of the load. Following that, the suitable formula can be used to determine the load bearing capacity of an eccentrically loaded stocky bar. In this analysis we didn't consider the case of the pentagonal compress zone.

\section{NUMERICAL EXAMPLES}

Check the load bearing capacity of the foundation with the following data in elastic and plastic state as well! We keep the same symbols and coordinate system, which are shown in Figure 3.

The loads - which act below the foundation - are shown in Figure 10.

$$
\begin{aligned}
& N=100 \mathrm{kN} \\
& M_{X}=30 \mathrm{kNm} \\
& M_{Y}=15 \mathrm{kNm}
\end{aligned}
$$

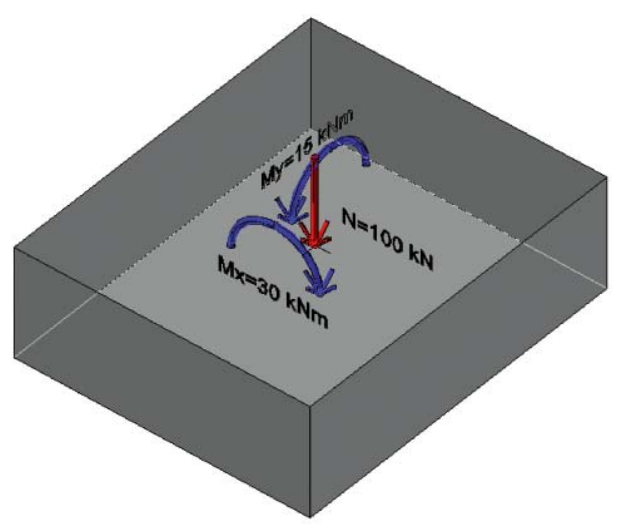

Figure 10 The loads of the foundation

Sizes of the cross section:

$$
\begin{aligned}
& a=1,0 m \\
& b=1,2 m
\end{aligned}
$$

The yielding compressive strength of the soil below the cross section:

$$
f_{y}=600 \frac{k N}{m^{2}}
$$


Calculation of the magnitudes of eccentricity:

$$
\begin{aligned}
& e_{X}=\frac{M_{X}}{N}=\frac{30}{100}=0,3 \mathrm{~m} \\
& e_{Y}=\frac{M_{Y}}{N}=\frac{15}{100}=0,15 \mathrm{~m}
\end{aligned}
$$

Calculation of the coordinates of the point of action:

$$
\begin{aligned}
& X_{S}^{\prime}=\frac{a}{2}-e_{X}=\frac{1,0}{2}-0,3=0,2 m \\
& Y_{s}^{\prime}=\frac{b}{2}-e_{Y}=\frac{1,2}{2}-0,15=0,45 m
\end{aligned}
$$

Analysis in elastic state:

First of all, we should determine whether the force causes a trapezoid compressed zone or a pentagonal one. Using Eq. (3) the length of the " $y$ " edge of the stress body can be calculated.

$$
\begin{aligned}
& y=\frac{-6 \cdot 1,2 \cdot 0,45+4 \cdot 1,2^{2}+1,2 \sqrt{-2 \cdot 1,2^{2}+12 \cdot 1,2 \cdot 0,45-12 \cdot 0,45^{2}}}{6 \cdot 1,2-12 \cdot 0,45} \\
& y=2,121 \mathrm{~m}
\end{aligned}
$$

Now, using Eq. (8) the " $X_{S}^{\prime \prime \prime}$ coordinate of the point of the curve can be determined, which belongs to the given " $Y_{S}^{\prime}$ " coordinate.

$$
X_{S}^{\prime}=\frac{1,0 \cdot\left(2 \cdot 2,121^{3}-3 \cdot 2,121^{2} \cdot 1,2+2 \cdot 2,121 \cdot 1,2^{2}-\frac{1,2^{3}}{2}\right)}{6 \cdot 2,121^{3}-6 \cdot 2,121^{2} \cdot 1,2+2 \cdot 2,121 \cdot 1,2^{2}}=0,263 \mathrm{~m}
$$

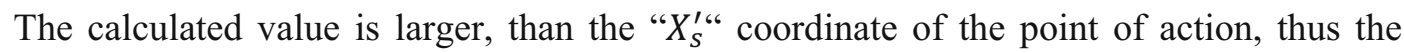
force acts outside of the curves, which means, that it causes a trapezoidal compressed zone. The point of action and the curve are shown in Figure 11. 


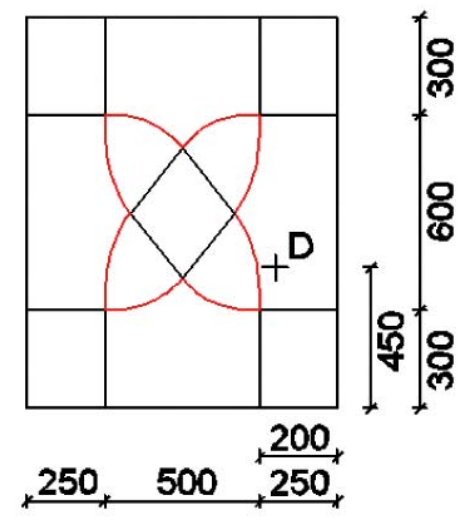

Figure 11 The point of action

Afterwards, the length of the edge into " $\mathrm{x}$ " direction of the stress body should be determined. For that, Eq. (5) can be used.

$$
x=\frac{6 \cdot 2,121^{3} \cdot 0,2-6 \cdot 2,121^{2} \cdot 1,2 \cdot 0,2+2 \cdot 2,121 \cdot 1,2^{2} \cdot 0,2}{2 \cdot 2,121^{3}-3 \cdot 2,121^{2} \cdot 1,2+2 \cdot 2,121 \cdot 1,2^{2}-\frac{1,2^{3}}{2}}=0,762 \mathrm{~m}
$$

With the knowledge of the sizes of the stress body the load bearing capacity of the stocky bar can be determined (in elastic state). We can use Eq. (6) for the calculation.

$$
V=\frac{1,2 \cdot 0,762 \cdot 600}{2} \cdot\left(1-\frac{1,2}{2,121}+\frac{1,2^{2}}{3 \cdot 2,121^{2}}\right)=148,39 k N
$$

Thus, the elastic load bearing capacity of the foundation is $148,39 \mathrm{kN}$.

$$
V=148,39 k N>N=100 k N
$$

Thus, the foundation is satisfactory!

Analysis in plastic state:

We can use the same method, which we used in elastic state. At the beginning we should analyse whether the point of action is located in the green or the red shaded area in Figure 7. For the determination of the length of the edge into " $y$ " direction of the stress body Eq. (10) can be used.

$$
y=\frac{2 \cdot 1,2^{2}-3 \cdot 1,2 \cdot 0,45}{3 \cdot 1,2-6 \cdot 0,45}=1,4 m
$$


After that, with a substitution into the Eq. (15) the " $X_{S}^{\prime \prime c}$ coordinate of the point of the curve can be determined, which belongs to the given " $Y_{S}^{\prime}$ " coordinate.

$$
X_{s}^{\prime}=\frac{1,0 \cdot\left(1,2^{2}-3 \cdot 1,2 \cdot 0,45+3 \cdot 0,45^{2}\right)}{2 \cdot 1,2^{2}-3 \cdot 1,2 \cdot 0,45}=0,339 \mathrm{~m}
$$

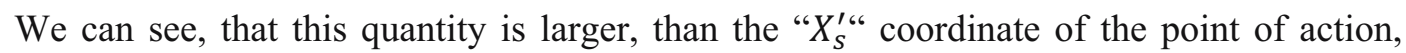
thus the force acts outside of the curves, which means, that it causes a trapezoidal compressed zone. The point of action and the curve are shown in Figure 12.

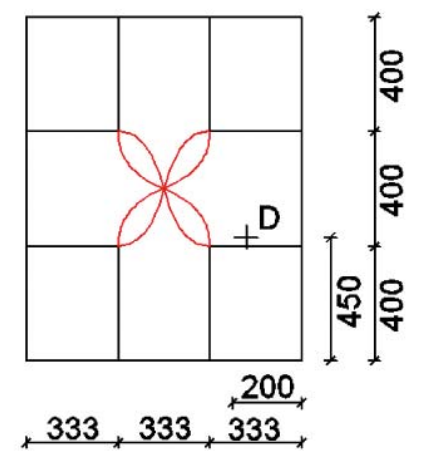

Figure 12 The point of action

Afterwards, using Eq. (12) the length of the "x" edge of the stress body can be calculated.

$$
x=\frac{2 \cdot 0,2 \cdot 1,4^{2}-0,2 \cdot 1,2 \cdot 1,4}{1,4^{2}-1,2 \cdot 1,4+\frac{1,2^{2}}{3}}=0,589 \mathrm{~m}
$$

Thus, we determined the sizes of the stress body. Therefore, we can calculate the load bearing capacity of the stocky bar can be calculated (in plastic state). For this calculation Eq. (13) can be used.

$$
V=600 \cdot 1,2 \cdot 0,589 \cdot\left(1-\frac{1,2}{2 \cdot 1,4}\right)=242,33 \mathrm{kN}
$$

Thus, the plastic load bearing capacity of the stocky bar is $242,33 \mathrm{kN}$.

$$
V=242,33 k N>N=100 k N
$$

Thus, the foundation is satisfactory!

\section{AcKnOWLEDGments}

I would like to say thank you to Dr. János Fischer for the great support and inspiration. 


\section{REFERENCES}

[1] Gere and Timoshenko, Mechanics of Materials, Chapmen and Hall, London 1991. p. 298.

[2] Mutrnyánszky, Á., Szilárdságtan, Műszaki Könyvkiadó, Budapest: 1981. p. 294.

[3] Tóвı́́s. L., Épületszerkezetek Szilárdságtana, Budapest 1947. p. 136.

[4] Pelikán, J., Szilárdságtan, Tankönyvkiadó, Budapest 1972. p. 136.

[5] Сноцnoky, T., Mechanika II., Tankönyvkiadó, Budapest 1966. p. 449.

[6] Bárczi, I., Falu Gy., Zalka K., Mechanika II. Szilárdságtan, Tankönyvkiadó, Budapest: 1989. p. 228.

[7] BÁRCZI, I., Teherhordó szerkezetek zsebkönyve, Müszaki Könyvkiadó, Budapest: 1984. p. 232.

[8] Becker, S., Szilárdságtan I., Nemzeti Tankönyvkiadó, Budapest: 1989. p. 132. 